\title{
Post-analysis of the Swine Flu Pandemic: Overreaction or Necessary Precaution?
}

\section{By Inderjeet Sahota}

In April 2009 news reports began to surface of an influenza-like outbreak in Mexico. Mexican officials did whatever they could to curb its spread but the world watched anxiously as their efforts seemed insufficient in preventing further outbreaks ${ }^{1}$. Cases of swine flu, as it was now being referred to, were emerging in cities all across North America, Europe and Asia. Just two months after the initial reports in Mexico the World Health Organization (WHO) would declare this swine flu a pandemic and issue protocols in the hope that it would stop further spread. One after another, countries began declaring this virus a national priority and pharmaceutical companies dedicated themselves to finding a vaccination. News reports tirelessly warned us of the immediate threat this virus posed to health and a genuine sense of paranoia ensued as people became too scared to be in public places. different.

Just over a year later the situation is very

You'd be hard pressed to find a news report on the swine flu now. So, what happened? As the dust begins to settle, health officials are beginning to ask themselves whether the global reaction to swine flu was necessarily precautionary or simply an overreaction. Around 12,700 people worldwide died from H1N1, an unfortunately sizable number ${ }^{2}$. However, this value needs to be taken into context. According to the Centre for Disease Control in the United States, about 36,000 people die from seasonal flu-related causes each year in the United States alone ${ }^{3}$. This does not necessarily mean the flu was the primary cause of death but even so, the numbers indicate how relatively small the deaths from H1N1 were in comparison to the number of people that die from the seasonal flu each year. Also, even before mass vaccination programs were introduced most people infected with H1N1 were able to recover within weeks with no long-term complications. Although the physical loss from H1N1 remains fortunately small, the economic cost

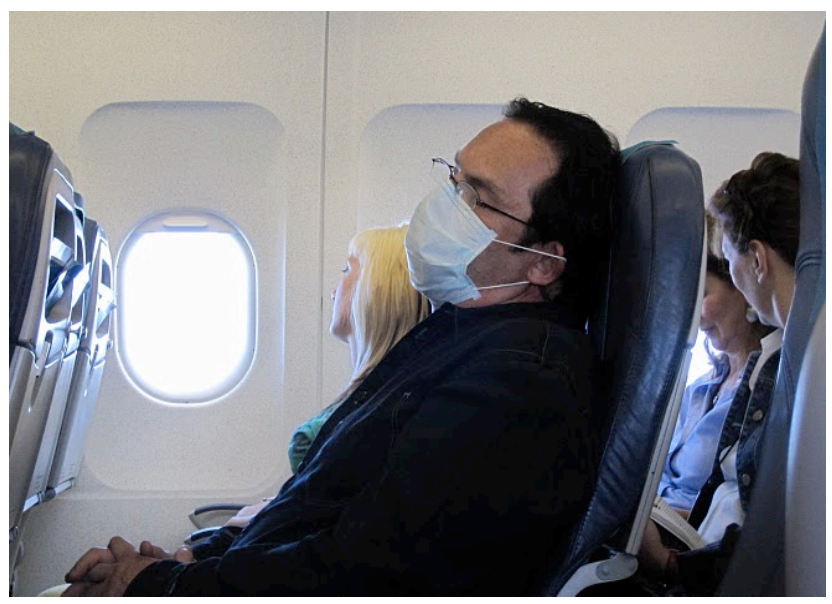

may not be so minute. The final global cost of the pandemic has yet to be determined. However, analysts believe it will likely amount to billions of dollars ${ }^{2}$. Wealthier countries have already confirmed to have spent that much on medicines and vaccinations alone and many governments are now trying to resell their stockpiles of the swine flu vaccine.

The WHO is now under scrutiny following accusations that they may have exaggerated the pandemic to bring economic benefits to the pharmaceutical industry. Although this is unlikely, the allegations were serious enough to warrant the WHO to release an official statement on January 22, 2010 addressing the matter ${ }^{4}$. As months go by, it will be interesting to witness the results of an independent investigation of the management of the H1N1 pandemic and this might elucidate whether the global response was appropriate or grossly disproportionate.

The swine flu vaccinations themselves offer another potential problem. In Canada, and much of the rest of the world, there have been serious questions regarding the safety of the H1N1 vaccinations that were administered. As the need for deployment was paramount, pharmaceutical companies had a limited time to administer these 
vaccinations around the world. As such, appropriate long-term testing was deferred as vaccinations were fast-tracked through the process in an effort to curb this influenza pandemic. It remains to be seen whether there are any long-term effects of these vaccinations. Unfortunately, as many people have already undergone the treatment, the first results we have may be from case reports, not laboratory reports.

So, was the global response an over-reaction or a necessary precaution? I believe that the overall response was in the right direction. Many health officials echo the concerns listed above but ultimately understand that the risk of this viral strain killing millions was worth the swift response ${ }^{5}$. However, I feel that although the response was in the right direction it wasn't the right magnitude. If health officials were able to determine early on that this influenza strain was of relatively moderate virulence, then an action plan that better suited the situation, rather than a seemingly all-or-nothing reaction, may have been more efficient. A tiered response where global protocols are issued according to an accurate level of severity would probably be best when dealing with future pandemics. In the next year it will be interesting to see what the WHO and other governmental organizations determine from their analysis of the response and the changes that can be applied for similar emergencies in the future.

\section{References}

1. Mexico imposes swine flu measures. BBC News. Available online: http://news.bbc.co.uk/2/hi/americas/8023820.stm

2. World Health Organization to review swine flu response. BBC News. Available online: [http://news.bbc.co.uk/2/hi/health/8455035.stm].

3. Estimating Deaths from Seasonal Influenza in the United States. Centers for Disease Control and Prevention. Available online: [http://www.cdc.gov/flu/about/disease/us_flurelated_deaths.htm]

4. Statement of the World Health Organization on allegations of conflict of interest and 'fake' pandemic. World Health Organization. Available online: [http://www.who.int/mediacentre/news/statements/2010/h 1n1_pandemic_20100122/en/index.html]

5. Did UK get it right on swine flu? BBC News. Available online: [http://news.bbc.co.uk/2/hi/health/8458668.stm]

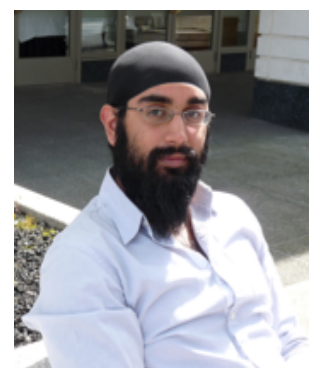

\section{Author Profile}

Inderjeet Sahota is currently a MSc candidate from the Department of Biomedical Physiology and Kinesiology at Simon Fraser University. His main interests in research involve understanding cardiovascular and cerebrovascular alterations that occur in spinal cord injury (SCI). The purpose of my project is to determine the changes in cerebrovascular blood flow velocity (CBFV), as well as other cardiovascular parameters, that occur in people with spinal cord injury during changes in hypo- and hypertensive situations (during orthostatic hypotension and autonomic dysreflexia respectively). We hope that by understanding the changes that occur in CBFV we may help confer better treatment protocols for the future. Inderjeet is also currently serving as Health Science Inquiry's Managing Editor. 